\title{
IMPACTO DE LA ADICIÓN DE CABOXIMETILCELULOSA Y AGUA EN LAS PROPIEDADES FISICOQUÍMICAS Y DE CALIDAD DE PAN LIBRE DE GLUTEN
}

\section{IMPACT OF CARBOXYMETHYLCELLULOSE AND WATER ADDITION ON BAKING QUALITY AND PHYSICOCHEMICAL PROPERTIES OF GLUTEN-FREE BREAD}

\author{
Fernán Martínez-Jiménez ${ }^{1}$, Eduardo Rodríguez-Sandoval², María Soledad Hernández-Gómez ${ }^{3}$
}

\begin{abstract}
${ }^{1}$ Ingeniero de Alimentos, Departamento Ingeniería Agrícola y Alimentos, Facultad de Ciencias Agrarias. Universidad Nacional de Colombia, calle 59A No 63-20, Medellín, Antioquia, Colombia, e-mail: fernandavidm@gmail.com; ${ }^{2}$ Doctor en Ingeniería, Profesor Asociado, Departamento Ingeniería Agrícola y Alimentos, Facultad de Ciencias Agrarias. Universidad Nacional de Colombia, calle 59A No 63-20, Medellín, Antioquia, Colombia, e-mail: edrodriguezs@unal.edu.co; ${ }^{3}$ Doctor en Ciencias Agrarias, Profesora Titular, Instituto de Ciencia y Tecnología de Alimentos (ICTA). Universidad Nacional de Colombia, carrera 45 No 26-85, Bogotá D.C., Colombia, e-mail: mshernandez@unal.edu.co
\end{abstract}

Rev. U.D.C.A Act. \& Div. Cient. 18(2): 445-454, Julio-Diciembre, 2015

\section{RESUMEN}

El objetivo de este estudio fue determinar la influencia de la adición de agua y de carboximetilcelulosa (CMC) en las propiedades fisicoquímicas y de calidad de pan libre de gluten (GF), elaborado con harina de arroz, almidón de maíz y almidón de yuca. La harina de arroz presentó los valores más altos de índice de absorción de agua (WAI) e índice de solubilidad de agua (WSI). Las concentraciones de CMC y agua fueron de $1-3 \%$ y $80-90 \%$, con base en la harina de arroz, componente mayoritario de la mezcla, respectivamente. Se utilizó la Metodología de Superficie de Respuesta (MSR). Los valores máximos para el volumen específico $(3,92 \mathrm{~mL} / \mathrm{g})$ y la altura $(77,82 \mathrm{~mm})$, se mostraron con $85 \%$ de agua y $2 \%$ de CMC. El mayor rendimiento, se evidenció con 3\% de CMC y $80 \%$ de agua, pero este tratamiento tuvo el volumen específico y la altura más baja. En cuanto a las propiedades texturales, la combinación de 3\% de CMC y $80 \%$ de agua generó una miga con la mayor firmeza $(16,6 \mathrm{~N})$ y dureza $(91,2 \mathrm{~N})$; los valores más bajos de estos parámetros, se presentaron con $85 \%$ de agua y $2 \%$ de CMC. El mayor valor de cohesividad $(0,46)$, se registró con $90 \%$ de agua y $3 \%$ de CMC y la condición más baja de cohesividad, se reportó con $80 \%$ de agua y $1 \%$ de CMC. La inclusión de mayores concentraciones de CMC (3\%) genera productos GF, con mayor firmeza, cohesividad, elasticidad y dureza.

Palabras clave: Celiaquía, almidón, hidrocoloide, textura, entalpia.

\section{SUMMARY}

The objective of this study was to determine the influence of water and carboxymethylcellulose (CMC) addition on physicochemical properties and baking quality of gluten-free (GF) bread made from rice flour, corn and cassava starch. Rice flour showed the highest values of water absorption index (WAI) and water solubility index (WSI). The CMC concentrations and moisture content were $1-3 \%$ and $80-90 \%$, based on rice flour, major component of the mixture, respectively. Response Surface Methodology (RSM) was used. The maximum values for the specific volume $(3.92 \mathrm{~mL} / \mathrm{g})$ and height $(77.82 \mathrm{~mm})$ are presented with $85 \%$ moisture content and $2 \%$ CMC. The best yields were obtained with $3 \%$ CMC and $80 \%$ moisture content; but this treatment had the lowest specific volume and height. As for the textural properties, the combination of $3 \%$ CMC and $80 \%$ moisture content resulted in higher firmness crumb (16.6N) and hardness (91.2N), the lowest values of these parameters were presented with $85 \%$ moisture content and 2\% CMC. The higher cohesiveness value (0.46) was showed with $90 \%$ moisture content and $3 \%$ CMC, and lower cohesiveness condition reported with $80 \%$ moisture content and $1 \% \mathrm{CMC}$. The adding of higher concentrations of CMC (3\%) resulted in GF products with more firmness, cohesiveness, elasticity and hardness.

Key words: Celiac disease, starch, hydrocolloid, texture, enthalpy. 


\section{INTRODUCCIÓN}

El incremento de la demanda de los productos libres de gluten, se debe al aumento real o apartente de la enfermedad celiaca o a otras reacciones alergicas al consumo de gluten. La enfermedad está relacionada con la inflamación del intestino delgado, lo que conlleva a la mala absorción de varios nutrientes importantes y al daño de la mucosa intestinal. El único tratamiento efectivo es una dieta estricta, sin gluten durante toda la vida (Lazaridou et al. 2007; Arendt et al. 2008). La sustitución de la matriz de gluten en los procesos de panificación representa un desafío tecnológico, dado que atribuye características reológicas deseables al pan, como la elasticidad, la extensibilidad, la resistencia a la deformación y la capacidad de retención de gas. Las masas sin gluten -antes del horneo- presentan mucha fluidez, ocasionando características indeseables, como miga débil, fisuras en la corteza y otros defectos de calidad (Lazaridou et al. 2007; Goesaert et al. 2005; Arendt et al. 2008). Además, la mayoría de los productos de panificación sin gluten que se consiguen en el mercado son de baja calidad, por tal motivo, se ha buscado un sustituto al gluten, para la fabricación de este tipo de productos. Para su producción, se necesita, principalmente, almidones, proteínas (lácteas, soya u otras) y sustancias poliméricas, que imiten las propiedades viscoelásticas del gluten (Arendt et al. 2008).

En productos de panificación, el almidón contribuye a la textura, a la apariencia y a la aceptabilidad general de los alimentos a base de cereales. Los hidrocoloides son polímeros hidrófilos de origen vegetal, animal, microbiano o de material sintético que, por lo general, contienen gran cantidad de grupos hidroxilos y son ampliamente utilizados para controlar las propiedades funcionales de los alimentos. Los hidrocoloides han sido ampliamente utilizados, como aditivos para mejorar la textura de los alimentos y las características viscoelásticas, reducir la velocidad de retrogradación del almidón, actuar como aglutinante de agua; aumentar la vida útil y funcionan como sustitutos del gluten en procesos de panificación (Arendt et al. 2008).

El aumento en la concentración de metilcelulosa (MC), de carboximetilcelulosa (CMC) o de hidroxipopilmetilcelulosa (HPMC) aumenta la firmeza de la miga. El proceso de producción de estos hidrocoloides, se realiza mediante la sustitución de hidrógeno en el grupo hidroxilo de la celulosa con metiléter, carboximetílico o grupos hidroxipropilo, en condiciones alcalinas. La celulosa modificada produce polímeros solubles en agua, con una afinidad por la fase no polar de la masa; por consiguiente, la afinidad neta de las fases acuosa y no acuosa de la masa ayuda a mantener la uniformidad y la estabilidad de la emulsión de la masa. Esto induce propiedades adicionales, como un aumento de la actividad interfacial en los límites de las alveolos de gas en expansión, lo que resulta en masas más firmes (Onyango et al. 2009b).

Los panes libres de gluten elaborados a partir de harina de arroz, metilcelulosa (MC) y agar-agar presentan alta porosidad y miga no homogénea, mientras que los preparados con goma xantana, CMC, xantana -guar, xantana -algarrobo e hidroxipropilmetilcelulosa (HPMC) muestran miga uniforme (Demirkesen et al. 2014). El uso de gomas xantana y guar,a partir de harina de arroz, de almidón de maíz y de papa, mejoró el rendimiento del producto; además, la mezcla de diferentes niveles de estas gomas, aumentó la retención de gas al interior de la matriz, ayudando a la estabilidad de la masa, durante el proceso de horneo (Mahmoud et al. 2013). El pan libre de gluten elaborado con almidón de maíz y con harina de arroz presentó mayor cantidad de alveolos al usar CMC, mientras que con goma xantana mostró una diminución en la dureza, un aumento en la elasticidad y una mayor capacidad para retener agua. Al mezclar los dos hidrocoloides, se consiguió una disminución en la dureza, aumento en la elasticidad y mayor retención de gas (Arendt et al. 2008).

Entre los almidones modificados, procedentes de almidón de yuca, utilizados en productos de panadería, se destaca la línea Expandex ${ }^{\circledR}$ de Ingredion Colombia S.A. El Expandex® es un ingrediente empleado en el mercado de productos libres de gluten, que tiene unas características de expansión únicas, similares a las encontradas en productos que contienen gluten. También, ayuda a retener agua, mejora las propiedades de la masa durante los ciclos de congelacióndescongelación y no contribuye a dar ningún sabor u olor particular. Entre los beneficios de la utilización de este ingrediente, se incluyen: mejorar la apariencia textura y sabor de los productos de panadería y repostería, generar una miga más húmeda y suave, reducir la cantidad de hidrocoloides necesarios en la formulación y aumentar la vida útil de los productos libres de gluten (Ingredion Colombia S.A., 2013).

Por otro lado, la formación de una red estable de proteína es crucial en la producción de panes libres de gluten (GF), con calidad aceptable. Fuera de mejorar la textura, los ingredientes ricos en proteína aumentan la calidad nutricional de los productos GF. Posibles ingredientes de proteína para la formulación de panes GF son extractos de plantas, como derivados de soya, aislados de garbanzo, sésamo y frijol. Los extractos animales, como concentrado de proteína de pescado, huevo o fracciones de leche, también pueden ser empleados; sin embargo, el uso comercial de algunas de estas proteínas, se puede restringir por varios factores, como disponibilidad, costo, fácil producción, presencia de inhibidores o compuestos que inducen alergias, intolerancia al consumo y propiedades funcionales limitadas. La soya, se puede utilizar en la formulación de productos GF en difer- 
entes formas, como harina con o sin grasa, concentrado o asilado (Onyango et al. 2009a). La soya contiene fitoquímicos que tienen beneficios a la salud como prevención del cáncer y ostoporosis, y reducción del colesterol. Además, la soya también exhibe actividad antioxidante natural, que puede aumentar la vida útil de los productos, a los que se les ha incorporado (Conforti \& Davis, 2006).

A pesar de los trabajos realizados en panes libres de gluten, a partir de harina de arroz, almidón de maíz, almidón de papa y diferentes combinaciones de estos materiales, aún no se cuenta con estudios de panes elaborados con almidón de yuca modificado (Expandex®), almidón de maíz y harina de arroz, usando carboximetilcelulosa (CMC). Por lo tanto, el objetivo de este estudio es determinar las propiedades texturales y de calidad de pan elaborado, a partir de harina de arroz, almidón de maíz y Expandex®, cambiando la concentración de hidrocoloide y agua en su formulación.

\section{MATERIALES Y MÉTODOS}

Para la formulación del pan libre de gluten, se emplearon harina de arroz blanco (Haritec, Alimentos Caribe, Itagüi, Colombia), almidón modificado de yuca Expandex® (Ingredion Colombia S.A., Cali, Colombia), almidón de maíz Buffalo (Ingredion Colombia S.A., Cali, Colombia), aislado de proteína de soya (Solae, Tecnas, Medellín, Colombia), carboximetilcelulosa sódica de grado alimenticio (Protokimica, Medellín, Colombia), ésteres de ácido diacetil tartárico de monoglicéridos (DATEM) (Panodan G 20-20, Danisco), azúcar, sal, levadura instantánea, margarina, propionato de calcio y agua. El contenido de humedad de la materia prima (almidón modificado de yuca, harina de arroz, almidón de maíz y aislado de proteína de soya) fue determinado secando $2 \mathrm{~g}$ de muestra en un horno (UFE-400, Memmert GmbH + Co. KG, Munich, Alemania), de acuerdo al método 44-15.02 AACC (AACC, 2000). El contenido total de nitrógeno de la materia prima, se evaluó de acuerdo al método 920.87 AOAC (AOAC, 1997); el contenido de proteína fue calculado adoptando 6,25, como factor de conversión. Grasa (945.16), ceniza (942.05) y fibra cruda (978.10), se establecieron usando la metodología de AOAC (AOAC, 1997). El contenido de almidón, se determinó por el método polarímetro de Ewers (ISO 10520, 1997). Todas las mediciones, se realizaron por duplicado.

Las propiedades térmicas de las muestras amiláceas, se monitorearon usando un DSC (DSC-Q200, TA Instruments, New Castle, DE, USA); la calibración, se realizó con indio y una cápsula vacía, que se usó como referencia. La muestra, se selló con una prensa, se dejó equilibrar durante $24 \mathrm{~h}$ y se calentó de $25^{\circ} \mathrm{C}$ hasta $100^{\circ} \mathrm{C}$, empleando una rampa de calentamiento de $10^{\circ} \mathrm{C} / \mathrm{min}$ y una rampa de enfriamiento de $25^{\circ} \mathrm{C} / \mathrm{min}$, hasta llegar a la temperatura inicial (Rodriguez et al. 2001). Los resultados fueron: temperatura de inicio (To), temperatura de pico $\left(T_{p}\right)$, temperatura final $\left(T_{f}\right)$ y cambio en la entalpia de fusión o de gelatinización $(\Delta \mathrm{H})$. Esta medición, se realizó por triplicado.

Las pruebas de índice de absorción de agua (WAI) e índice de solubilidad de agua (WSI), se pueden utilizar como un indicativo del grado de modificación de los almidones por tratamientos termomecánicos. El método que se siguió para la determinación de WAI y WSI fue tomado de Anderson et al. (1969), con algunas modificaciones. Las pruebas de WAI y WSI, se determinaron pesando $0,65 \mathrm{~g}$ de muestra y $7,8 \mathrm{~g}$ de agua destilada en un tubo de centrifuga, empleando una balanza de precisión. Después, el tubo con la muestra, se llevó a un vortex (Classic advanced, Velp scientifica, Usmate, Italia), durante $30 \mathrm{~s}$; la muestra, se colocó en baño maría, con agitación (WNB-14, Memmert GmbH + Co. KG, Munich, Alemania), durante $30 \mathrm{~min}$, a $30^{\circ} \mathrm{C}$; luego, se centrifugó (Universal 320-R, Andreas Hettich GmbH \& Co. KG, Tuttlingen, Alemania), durante $10 \mathrm{~min}$, a $5200 \mathrm{rpm}$ y $20^{\circ} \mathrm{C}$. El sobrenadante de las muestras centrifugadas, se adicionó a una caja Petri y el sedimento permaneció en el fondo del tubo. Ambas muestras se pesaron. El sobrenadante se secó a $105^{\circ} \mathrm{C}$, durante $24 \mathrm{~h}$ en estufa. Las muestras, se realizaron por triplicado.

En la elaboración del pan, inicialmente, se mezclaron las harinas: $250 \mathrm{~g}$ de harina de arroz, $125 \mathrm{~g}$ de almidón modificado de yuca y $125 \mathrm{~g}$ de almidón de maíz, durante $2 \mathrm{~min}$, en una batidora (Profesional Series 600-KP26M1XER, Kitchenaid, St. Joseph, MI, USA). Después, se incorporó $30 \mathrm{~g}$ de aislado de proteína de soya, $25 \mathrm{~g}$ de azúcar, $10 \mathrm{~g}$ de sal, $2,5 \mathrm{~g}$ de propionato de calcio, $2,5 \mathrm{~g}$ de DATEM y CMC, de acuerdo al diseño experimental; luego, se disolvió $15 \mathrm{~g}$ de levadura en $150 \mathrm{~g}$ de agua, se adicionó a la mezcla con $40 \mathrm{~g}$ de margarina y se mezcló durante 2 min más; por último, se adicionó el agua restante, de acuerdo al diseño experimental y se mezcló por 4 min, hasta obtener una masa homogénea. El moldeado, se realizó en recipientes de aluminio engrasados $(17,2 \mathrm{~cm}$ de largo, $6,2 \mathrm{~cm}$ de alto, $8,9 \mathrm{~cm}$ de ancho), donde se depositó -en forma uniforme- $280 \mathrm{~g}$ de masa. Los moldes con masa, se situaron en un fermentador, a $35-38^{\circ} \mathrm{C}$, con una humedad relativa por encima de $80 \%$, durante $30 \mathrm{~min}$. Las masas fermentadas, se hornearon en un horno de gas (GFO-4B, Guangzhou Youjia Machinery Co, China), a $200^{\circ} \mathrm{C}$, durante 25 min.

El porcentaje de rendimiento del proceso, se determinó empleando la relación de la masa antes de fermentar y el producto después de horneado y enfriado. La altura del pan, se determinó realizando un promedio de las alturas de los extremos y de la mitad de la muestra; las medidas, se realizaron empleando un calibrador digital; el volumen específico del pan , se determinó usando la metodología de desplazami- 
ento de semilla de mijo (Sahin \& Sumnu, 2006); la humedad de la corteza y la miga ( $2 \mathrm{~g}$ de muestra), se determinó por secado en estufa, a $130^{\circ} \mathrm{C}$, durante $3 \mathrm{~h}$ (AOAC, 1997); la actividad de agua , se determinó empleando un higrómetro de punto de rocío, a $25^{\circ} \mathrm{C}$ (Aqualab serie 3TE, Decagon, Devices, Pullman, WA, USA) (Manjarres-Pinzon et al. 2013).

La firmeza de la miga, se midió empleando un analizador de textura (TA-XT2i, Stable Micro Systems, Godalming, U.K.), con celda de carga de $25 \mathrm{~kg}$, software TextureExpertExceed versión 2,64 (2002) y una sonda cilíndrica de 35mm (SMSP/35) y en modo fuerza en compresión. La prueba, se realizó empleando una rodaja de pan de $25 \mathrm{~mm}$ de grosor. Los parámetros de trabajo para la prueba fueron: velocidad de pre-test de $1,0 \mathrm{~mm} / \mathrm{s}$; velocidad de test de $1,7 \mathrm{~mm} / \mathrm{s}$; velocidad post-test de $10 \mathrm{~mm} / \mathrm{s}$ y compresión del $40 \%$ de la muestra $(10 \mathrm{~mm})$. La firmeza de la miga, se midió a una deformación del $25 \%$ de la muestra, de acuerdo al método AACC 74-09 (AACC, 2000).

La cohesividad, la dureza y la elasticidad de las muestras, se determinaron empleando el mismo analizador de textura, descrito, inicialmente, con una celda de carga de $50 \mathrm{~kg}$, un disco plano de $75 \mathrm{~mm}$ de diámetro (SMSP/75) y la prueba en modo TPA (Texture Analysis Profile). La muestra fue una rodaja de pan de $25 \mathrm{~mm}$ de grosor y los parámetros de trabajo fueron: velocidad de pre-test de $2 \mathrm{~mm} / \mathrm{s}$; velocidad de test de $2 \mathrm{~mm} / \mathrm{s}$; velocidad post-test de $5 \mathrm{~mm} / \mathrm{s}$; una compresión de $15 \mathrm{~mm}$ de la muestra y tiempo de espera $5 \mathrm{~s}$.

Los datos experimentales, se analizaron mediante un análisis de varianza (ANOVA), con el objetivo de conocer el efecto del CMC y agua sobre las características físicas y texturales del pan. Para el manejo de los datos, se empleó el software Design Expert versión 8.0.6 (Stat-Ease, U.S.A., 2010), aplicando la metodología de superficie de respuesta (RSM) y un diseño central compuesto (CCD), centrado frontal con 8 corridas sencillas (puntos factoriales y axiales) y 5 puntos centrales, con un nivel de significancia del $5 \%$ y de dos factores, $\%$ de CMC y $\%$ de agua, variando de $1 \%$ a $3 \%$ y de $80 \%$ a $90 \%$, respectivamente. Para establecer el ajuste del modelo, se observaron los coeficientes de determinación, la falta de ajuste y los gráficos de los residuos.

\section{RESULTADOS Y DISCUSIÓN}

La prueba de WAI mide el volumen ocupado por el gránulo de almidón después del hinchamiento en exceso de agua, mientras que la prueba de WSI determina la cantidad de moléculas libres lixiviadas del granulo de almidón en exceso de agua (Rodriguez-Sandoval et al. 2014). La tabla 1 presenta los valores de WAI, WSI y la composición química del almidón de maíz (CS), almidón de yuca modificado (E), aislado de proteína de soya (SPI) y harina de arroz (RF). Los valores más altos de WAI y WSI, se registraron en el aislado de proteína de soya y la harina de arroz, respectivamente. Por otro lado, el almidón de yuca modificado y de maíz mostraron los valores más bajos de WAI y WSI. Valores altos de WAI y WSI indican gelatinización del almidón, daños en su estructura y en su cristalización (Rodriguez-Sandoval et al. 2012). Kaushal et al. (2012) y Pongjaruvat et al. (2014) reportan valores semejantes a los encontrados en harina arroz de WAI y WSI $(2,43 \mathrm{~g} / \mathrm{g}$ y $2,66 \%$, respectivamente). Lee et al. (2008) encontraron un WAI de $1,96 \mathrm{~g} / \mathrm{g}$, para el almidón de yuca; sin embargo, el WSI está por encima de lo obtenido en esta investigación, 76,3\%. Diop et al. (2011) reportaron que el WSI, para el almidón de maíz, está por debajo de 0,5\% y el WAI, se encuentra en el rango de 2,25 a $2,5 \mathrm{~g} / \mathrm{g}$, lo cual, concuerda con lo presentado en esta investigación. De los materiales amiláceos empleados, la harina de arroz fue la que mostró el mayor contenido de proteína, de fibra cruda y de grasa, mientras que el almidón de maíz y de yuca modificado son las materias primas con mayor cantidad de almidón. El aislado de proteína de soya evidencia la mayor concentración de proteína y cenizas.

Tabla 1. Índice de absorción de agua (WAI), índice de solubilidad en agua (WSI) y composición química de la materia prima (promedio \pm SD).

\begin{tabular}{|l|c|c|c|c|}
\hline \multicolumn{1}{|c|}{ Propiedades } & CS* & $\mathrm{E}^{*}$ & RF$^{*}$ & SPI* \\
\hline Humedad (\%) & $11,7 \pm 0,3$ & $12,4 \pm 0,3$ & $9,3 \pm 0,3$ & $4,1 \pm 0,2$ \\
\hline $\begin{array}{l}\text { Cenizas }(\mathrm{g} / 100 \mathrm{~g} \\
\mathrm{dm})\end{array}$ & $0,1 \pm 0,0$ & $0,7 \pm 0,0$ & $0,7 \pm 0,1$ & $3,9 \pm 0,2$ \\
\hline $\begin{array}{l}\text { Fibra cruda } \\
\text { (g/100g dm }\end{array}$ & $0,1 \pm 0,0$ & $0,2 \pm 0,0$ & $0,3 \pm 0,0$ & $0,4 \pm 0,0$ \\
\hline $\begin{array}{l}\text { Grasa }(\mathrm{g} / 100 \mathrm{~g} \\
\text { dm) }\end{array}$ & $1,27 \pm 0,0$ & $0,14 \pm 0,0$ & $1,31 \pm 0,0$ & $0,41 \pm 0,0$ \\
\hline $\begin{array}{l}\text { Proteína }(\mathrm{g} / 100 \mathrm{~g} \\
\text { dm) }\end{array}$ & $0,6 \pm 0,0$ & $0,2 \pm 0,1$ & $8,0 \pm 0,1$ & $82,9 \pm 0,2$ \\
\hline $\begin{array}{l}\text { Almidón total } \\
\text { (g/100g dm) }\end{array}$ & $89,9 \pm 0,1$ & $91,0 \pm 0,1$ & $79,6 \pm 0,1$ & $1,1 \pm 0,0$ \\
\hline WAI $(\mathrm{g} / \mathrm{g})$ & $2,29 \pm 0,06$ & $1,99 \pm 0,02$ & $2,42 \pm 0,03$ & $7,62 \pm 0,03$ \\
\hline WSI (\%) & $0,25 \pm 0,02$ & $1,74 \pm 0,13$ & $2,06 \pm 0,03$ & -- \\
\hline
\end{tabular}

* La definición de cada sigla correspondiente a la muestra evaluada: CS: almidón de maíz, E: almidón de yuca modificado, SPI: aislado de proteína de soya, y RF: harina de arroz.

En cuanto a las propiedades térmicas, el almidón modificado de yuca presentó la entalpia más alta y la harina de arroz, la más baja, mientras que la temperatura inicial de gelatinización -para las tres muestras de almidones- empieza después de los $63^{\circ} \mathrm{C}$ (Tabla 2). La temperatura de pico 
más alta, se presentó en la harina de arroz y la menor, en el almidón modificado de yuca. Por último, la mayor temperatura final, se observó en el almidón modificado de yuca y la menor, en la harina de arroz. Las temperaturas de inicio, pico y final de gelatinización, para las muestras trabajadas, son semejantes a las reportadas en otras investigaciones (Hasjim et al. 2013; Hager et al. 2013). El almidón empieza a colapsar, estructuralmente, después de los $63^{\circ} \mathrm{C}$.

Tabla 2. Propiedades térmicas de materias primas amilaceas*.

\begin{tabular}{|c|c|c|c|}
\hline $\mathbf{T}_{0}\left[{ }^{\circ} \mathrm{C}\right]$ & $\mathbf{T}_{\mathrm{p}}\left[{ }^{\circ} \mathrm{C}\right]$ & $\mathrm{T}_{\mathrm{f}}\left[{ }^{\circ} \mathrm{C}\right]$ & $\Delta \mathbf{H}$ \\
\hline $63,90 \pm 0,34$ & $69,07 \pm 0,13$ & $79,98 \pm 0,08$ & $10,28 \pm 0,63$ \\
\hline $63,53 \pm 0,28$ & $69,04 \pm 0,66$ & $80,04 \pm 0,07$ & $12,76 \pm 0,71$ \\
\hline $63,27 \pm 0,30$ & $67,25 \pm 0,30$ & $82,09 \pm 1,96$ & $13,10 \pm 0,66$ \\
\hline
\end{tabular}

* La definición de cada sigla correspondiente a la variable respuesta: $T_{0}$ : temperatura de inicio, $T_{p}$ : temperatura de pico, $\mathrm{T}_{\mathrm{f}}$ temperatura final y $\Delta \mathrm{H}$ : cambio en la entalpia de fusión o de gelatinización.

La razón por la cual se presentan diferencias en las temperaturas y en las entalpias de gelatinización radica en la cantidad de almidón que tiene cada muestra, su estructura y composición (Ji et al. 2007). Según lo reportado por Hasjim et al. (2013), valores altos de temperatura de pico se dan por una alta resistencia a la transferencia de calor, lo que ocasiona que se deba aplicar más energía en forma de calor, para que los almidones se gelatinicen y ayuden al soporte del pan. En términos de calidad, la temperatura inicial de gelatinización indica la estabilidad térmica de la estructura molecular del almidón, por lo tanto, temperaturas bajas denotarían estructuras cristalinas modificadas. Al igual que la temperatura inicial, la entalpia de gelatinización tiene suma importancia en el análisis de calidad, dado que bajos valores de entalpia se dan por dos razones: el bajo contenido de almidón o una alta cantidad de almidón dañado (Onyango et al. 2011; Hasjim et al. 2013).

Los resultados obtenidos para el porcentaje de rendimiento, altura del pan, humedad de la corteza y miga, actividad de agua y volumen específico, se muestran en la tabla 3 . Respuestas obtenidas para cada corrida. La figura 1 (a), (b) y (c) presenta las respectivas superficies de respuesta para el rendimiento altura y volumen específico, las cuales, se ajustaron a modelos cuadráticos (Tabla 4). Con respecto a la altura, ninguno de los factores tuvo un efecto significante; sin embargo, para el rendimiento y el volumen específico, el único factor que presentó un efecto significante fue la cantidad de CMC. El tratamiento con $3 \%$ de CMC y $80 \%$ de agua reportó los valores más bajos en altura y volumen específico del pan. La razón de este comportamiento radica en la naturaleza hidrófila del hidrocoloide, que conlleva a una mayor retención de agua y un aumento de la viscosidad de la masa, produciendo una masa muy compacta y que crece muy poco en el proceso de leudado (Lazaridou et al. 2007). Otra posible razón es por la menor cantidad de agua empleada en la formulación, lo que ocasiona que la masa se vuelva quebradiza, se presenten defectos en la corteza y se deshidrate rápidamente la superficie (Arendt et al. 2008).

A medida que disminuye la concentración de CMC va disminuyendo el rendimiento, como se muestra en la figura 1(a). La presencia de fracturas en la corteza, durante el proceso de horneo, ocasionando que se escape gran cantidad de vapor, desde el interior de la matriz; una explicación para que suceda este comportamiento es por la disminución en la adición de CMC, lo cual, debilita la red estructural de la masa, aumentando la posibilidad de fracturas y mayor escape de agua, durante el horneo (Ziobro et al. 2013).

La presencia de fisuras en el pan es un indicativo de bajo volumen específico, ya que no se cuenta con una red que contenga el dióxido de carbono producido en la fermentación (Onyango et al. 2011; López-Tenorio et al. 2012). Los valores máximos de volumen específico y altura, se presentaron con $85 \%$ de agua y $2 \%$ de CMC, teniendo en cuenta la figura 1 (b) y (c), donde la retención de gas -en el interior de la matriz del alimento- se da por la formación de redes, que se originan por la interacción entre los derivados de celulosa (Onyango et al. 2009b). Esta estructura sirve para aumentar la viscosidad y fortalecer los límites de los alveolos en expansión, lo que facilita la retención de gas, a través del horneo y ocasiona un aumento en el volumen (Lazaridou et al. 2007). En diferentes estudios, se han encontrado que la adición de hidrocoloides, como el CMC, favorece la retención de gas en la matriz del pan, aumentando su volumen específico (Guarda et al. 2004; Lazaridou et al. 2007; Arendt et al. 2008; Hager et al. 2013). Adicionalmente, la proteína tiene un papel importante en la estabilidad del producto, ya que al desnaturalizarse proporciona un soporte a los almidones e hidrocoloides, ocasionando que el producto tenga características físicas deseables (Arendt et al. 2008; Ziobro et al. 2013). Por otro lado, el efecto de la composición de las materias primas (Tabla 1) y sus propiedades térmicas (Tabla 2) y funcionales, se ve reflejado en las características finales de los panes libres de gluten, elaborados de acuerdo a la formulación de este estudio.

Los datos obtenidos para la humedad de la corteza, de la miga y la actividad de agua no se ajustaron a ningún modelo matemático y, de igual forma, los factores no fueron significativos. La actividad de agua encontrada, experimentalmente, se encuentra dentro del rango $(0,95-0,98)$ y está de acuerdo 
Tabla 3. Respuestas obtenidas para cada corrida.

\begin{tabular}{|c|c|c|c|c|c|c|c|c|c|c|c|c|}
\hline \multirow{2}{*}{ Corrida } & \multicolumn{2}{|c|}{$\begin{array}{l}\text { Factores sin } \\
\text { codificar }\end{array}$} & \multicolumn{10}{|c|}{ Variables de respuesta* } \\
\hline & $\begin{array}{l}\text { Agua } \\
(\%)\end{array}$ & $\begin{array}{l}\text { CMC } \\
(\%)\end{array}$ & $\mathrm{R}[\%]$ & $\mathrm{h}[\mathrm{mm}]$ & $\mathrm{H}_{\mathrm{m}}[\%]$ & $\mathrm{H}_{\mathrm{c}}[\%]$ & $A_{w}[-]$ & $F_{m}[N]$ & $C[-]$ & $\mathrm{Du}[\mathrm{N}]$ & $\mathrm{E}[-]$ & $v$ \\
\hline 1 & 90 & 2 & 90,28 & 66,26 & 89,65 & 25,63 & 0,97 & 5,61 & 0,32 & 50,25 & 0,94 & 3,27 \\
\hline 2 & 90 & 1 & 88,65 & 58,45 & 85,25 & 21,21 & 0,97 & 5,80 & 0,29 & 64,88 & 0,85 & 2,55 \\
\hline 3 & 80 & 2 & 91,23 & 63,68 & 79,06 & 23,41 & 0,97 & 8,25 & 0,36 & 64,22 & 0,93 & 2,76 \\
\hline 4 & 85 & 2 & 89,86 & 69,61 & 82,81 & 25,57 & 0,97 & 3,58 & 0,37 & 46,81 & 0,90 & 2,93 \\
\hline 5 & 80 & 3 & 91,76 & 50,49 & 73,63 & 24,37 & 0,97 & 16,57 & 0,37 & 91,22 & 0,90 & 1,83 \\
\hline 6 & 85 & 2 & 87,81 & 77,82 & 77,46 & 19,63 & 0,97 & 2,29 & 0,39 & 41,81 & 0,95 & 3,10 \\
\hline 7 & 80 & 1 & 88,40 & 65,30 & 79,41 & 23,38 & 0,97 & 7,21 & 0,27 & 49,44 & 0,90 & 2,75 \\
\hline 8 & 85 & 2 & 89,44 & 62,57 & 77,47 & 25,10 & 0,97 & 4,86 & 0,41 & 52,60 & 0,92 & 3,05 \\
\hline 9 & 85 & 2 & 88,23 & 75,46 & 82,01 & 33,63 & 0,97 & 2,61 & 0,39 & 31,96 & 0,93 & 3,92 \\
\hline 10 & 85 & 3 & 91,29 & 59,41 & 82,68 & 31,46 & 0,96 & 8,39 & 0,44 & 82,18 & 0,94 & 2,31 \\
\hline 11 & 85 & 2 & 88,18 & 71,41 & 71,35 & 18,63 & 0,95 & 3,00 & 0,41 & 42,87 & 0,94 & 3,55 \\
\hline 12 & 85 & 1 & 87,07 & 59,27 & 72,48 & 21,53 & 0,95 & 5,65 & 0,32 & 53,90 & $0,8^{*}$ & 2,97 \\
\hline 13 & 90 & 3 & 91,21 & 58,48 & 84,88 & 30,87 & 0,96 & 7,78 & 0,47 & 44,35 & 0,93 & 1,95 \\
\hline
\end{tabular}

*La definición de cada sigla correspondiente a la variable respuesta: $\mathrm{R}$ rendimiento, $\mathrm{h}$ altura, $\mathrm{H}_{\mathrm{m}}$ humedad de la miga, $\mathrm{H}_{\mathrm{c}}$ humedad de la corteza, $\mathrm{A}_{\mathrm{w}}$ actividad de agua, $\mathrm{F}_{\mathrm{m}}$ firmeza de la miga, $\mathrm{C}$ cohesividad, Du dureza, E elasticidad, $v$ volumen especifico.

Tabla 4. Ecuaciones de los modelos determinados en las superficies de respuesta.

\begin{tabular}{|c|c|c|c|c|c|c|c|c|}
\hline \multirow{2}{*}{$\begin{array}{l}\text { Variable } \\
\text { respuesta }(Y)\end{array}$} & \multicolumn{6}{|c|}{ Coeficientes de regresión estimados } & \multicolumn{2}{|c|}{ Adecuación modelo } \\
\hline & & & & & & & $\mathbf{R}^{2}$ & $\begin{array}{l}\text { Falta de ajuste } \\
\quad(p>0,05)\end{array}$ \\
\hline $\mathrm{R}[\%]$ & 503,47 & $-9,88$ & 5,53 & 0,06 & $-0,12$ & $-0,04$ & 0,828 & 0,578 \\
\hline $\mathrm{h}[\mathrm{mm}]$ & $-965,89$ & 24,97 & $-27,53$ & $-0,15$ & $-9,50$ & 0,74 & 0,746 & 0,776 \\
\hline$F_{m}[N]$ & 856,07 & $-20,19$ & 21,31 & 0,12 & 3,11 & $-0,37$ & 0,939 & 0,257 \\
\hline $\mathrm{C}[-]$ & 11,63 & 0,28 & 0,28 & $-1,72 \mathrm{E}-3$ & $3,91 \mathrm{E}-3$ & $4,3 \mathrm{E}-3$ & 0,887 & 0,105 \\
\hline $\mathrm{Du}[\mathrm{N}]$ & 1010,24 & $-26,35$ & 211,63 & 0,18 & 15,37 & $-3,12$ & 0,820 & 0,237 \\
\hline $\mathrm{E}[-]$ & 5,21 & 0,03 & $-0,24$ & $-2,13 E-4$ & $-0,03$ & $4,3 \mathrm{E}-3$ & 0,776 & 0,773 \\
\hline$v$ & $-92,94$ & 2,23 & 1,11 & $-0,01$ & $-0,71$ & 0,02 & 0,809 & 0,887 \\
\hline
\end{tabular}



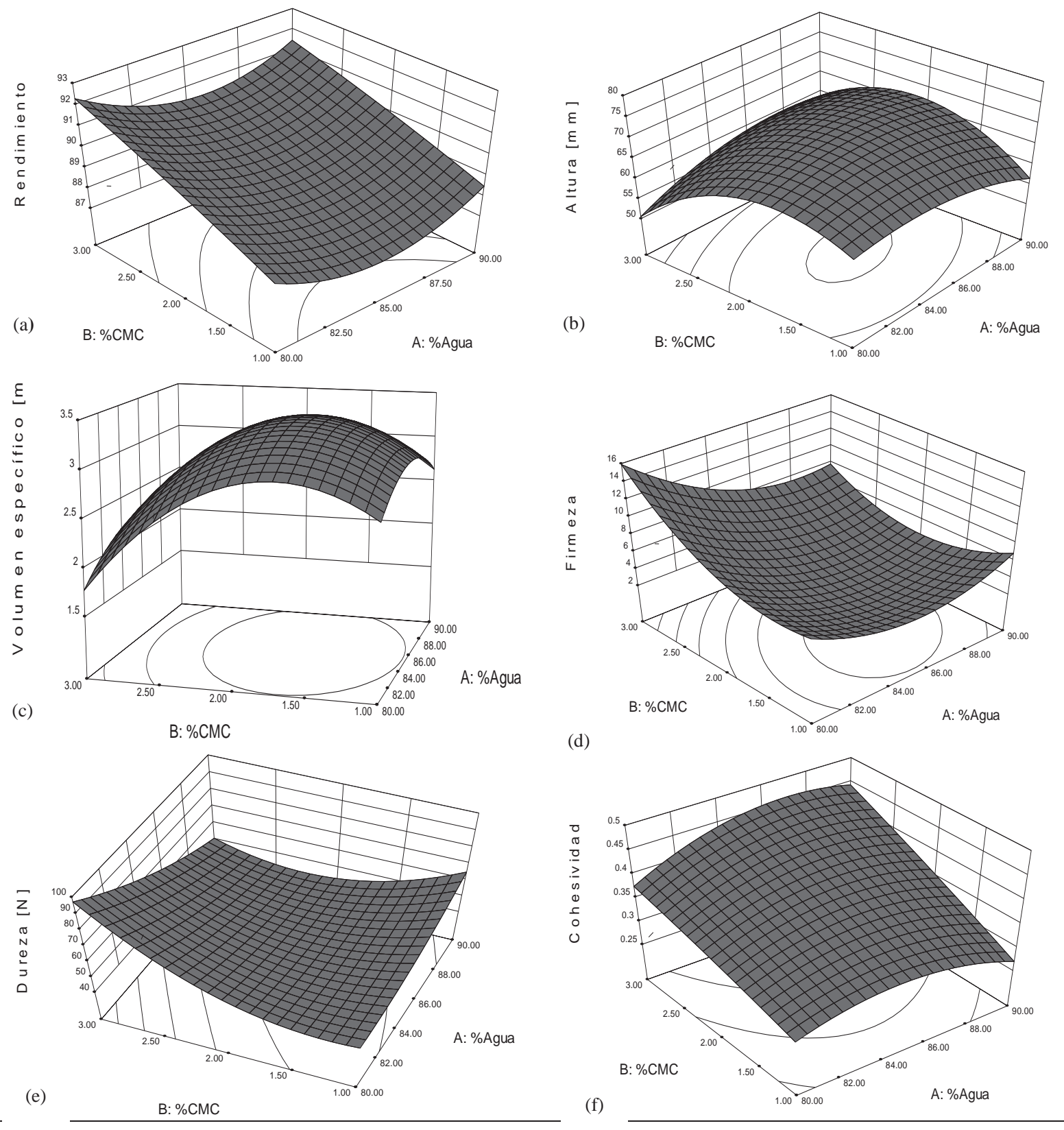

Figura 1 Propiedades físicas y texturales de pan libre de gluten. (a) rendimiento, (b) altura, (c) volumen especifico, (d) firmeza de la miga, (e) dureza y (f) cohesividad.

a los resultados obtenidos en investigaciones de productos libres de gluten (Lazaridou et al. 2007; Mariotti et al. 2013). Los mayores contenidos de humedad de la miga, se presentaron cuando se adicionó mayor cantidad de agua (90\%) y concentraciones superiores al 2\% de CMC. Estudios previos han mostrado una relación directa entre la humedad de la miga y la cantidad de CMC, usados en la formulación. Esto se da por los grupos hidroxilos presentes en el CMC, que ligan fuertemente el agua y evitan que se pierda por evaporación, durante el proceso de horneo (Guarda et al. 2004; Mohammadi et al. 2013; Hager et al. 2013). 
La tabla 3 presenta los valores de firmeza de la miga, dureza, cohesividad y elasticidad de las muestras, para cada tratamiento. La figura 1 (d), (e) y (f) presenta las respectivas superficies de respuesta para firmeza de miga, dureza y cohesividad, ajustándose a un modelo cuadrático. Con respecto a la fuerza de la miga, todos los factores fueron significantes, al igual que la interacción entre éstos. En cuanto a la elasticidad, el porcentaje de CMC es significante, al igual que la interacción de cada factor. Los factores por separados no son significantes en la dureza de las muestras; sin embargo, la interacción si lo son y para la cohesividad, el único factor significante fue el porcentaje de CMC.

La mayor firmeza de la miga, se presentó con una concentración de 3\% en CMC y $80 \%$ de agua, siendo esta misma muestra la que reportó los menores valores de volumen específico y altura. Estos resultados concuerdan con los reportados por Lazaridou et al. (2007), donde a menor cantidad de agua la muestra presentaba un aumento en la firmeza de la miga. En diferentes estudios, se ha encontrado que la fuerza de la miga disminuye a medida que aumenta el contenido de CMC y agua (Mohammadi et al. 2013; Sciarini et al. 2012; Demirkesen et al. 2010); además, se ha registrado que la cantidad de CMC ocasiona un aumento en la fuerza de la miga. Estos resultados son semejantes a los reportados por Onyango et al. (2009b), quienes consiguieron que el aumento en la concentración de metilcelulosa (MC), carboximetilcelulosa (CMC) o hidroxipopilmetilcelulosa (HPMC) conlleva al aumento de la firmeza de la miga. Una posible explicación del efecto del CMC en las propiedades mecánicas y la estructura de pan libre de gluten es por un aumento de la rigidez, como resultado de la disminución del hinchamiento de los gránulos de almidón y la reducción del lixiviado de amilosa (Mohammadi et al. 2013). Cabe destacar que, según el modelo encontrado, la interacción que más afecta la firmeza de la miga es el CMC, seguido de la doble interacción de este factor y el agua (Tabla 4). Este último es el de menor impacto en la firmeza, porque los constituyentes de la masa, los diferentes almidones, aislado de proteína de soya, lípidos, hidrocoloides y carbohidratos, van a estar compitiendo por el agua disponible, teniendo en cuenta los valores reportados por WAI, lo que ocasiona que disminuyan las interacciones agua-CMC y, por consiguiente, se reduce el efecto de esta interacción en la fuerza de la miga (Cappa et al. 2013; de la Hera et al. 2014).

La mayor elasticidad de las muestras, se presentó con una concentración de CMC de $2 \%$ y $85 \%$ de agua; la menor, con $90 \%$ de agua y $1 \%$ de CMC. Estos resultados concuerdan con lo reportado en la literatura, donde describen que la mayor elasticidad se da con una concentración de $2 \%$ de CMC (Arendt et al. 2008; Lazaridou et al. 2007). El aumento en la elasticidad de la masa refleja el grado de unión entre los elementos estructurales, por lo tanto, valores altos de elasti- cidad implican una menor deformación o ruptura de la red de material compuesto (Onyango et al. 2009b).

La cohesividad aumenta con mayor concentración de CMC y agua (Figura 1 (f). Esta variable refleja la cohesión interna del material, lo que indica que un pan con alta cohesividad es deseable, ya que forma una masa, en lugar de desintegrarse durante el proceso de masticación, mientras que valores bajos de cohesividad, indican aumento de la susceptibilidad del pan a la fractura (Onyango et al. 2011). En otros estudios, se ha encontrado que la cohesividad del producto aumenta a medida que se incrementa la cantidad de agua en la formulación, entre el rango de $70-80 \%$ de agua, lo cual, es congruente con lo hallado en este estudio y, en valores superiores al $85 \%$ de agua, se reporta una disminución en la cohesividad (de la Hera et al. 2014). Esto se podría explicar, porque los materiales van a estar interactuando con el agua y algunos van a ligar más agua que otros, afectando la formación de la matriz necesaria en la estructura de la masa libre de gluten (Arendt et al. 2008). La mayor firmeza de la miga, se presentó con 3\% de CMC y $80 \%$ de agua y la menor, se registró con el nivel medio de $2 \%$ de CMC y $85 \%$ de agua. Como se puede observar, en la figura 1 (e), un aumento en la concentración de CMC ocasiona un gran incremento en la firmeza del producto; este resultado ya se ha reportado en otro estudio (Guarda et al. 2004). El CMC es el factor más influyente en la firmeza de la miga. Demirkesen et al. (2010) indican que la adición de CMC en productos libres de gluten es esencial para asegurar que los parámetros de calidad sean semejantes a productos con gluten. Según Hager et al. (2013), el agua no tiene un efecto significante, pero la interacción agua-CMC sí es significante; esto se da por la alta afinidad del CMC en ligar agua. Como se puede observar, en la figura 1, la firmeza de la miga tienden a aumentar a medida que la concentración de CMC aumenta, cuando se emplea un $80 \%$ de agua en la formulación; en el caso de la cohesividad, se mostró el pico máximo, con $3 \%$ de CMC y $87 \%$ de agua, después de este punto, con una mayor cantidad de agua, la cohesividad disminuyó; estos resultados están de acuerdo a los reportados por Onyango et al. (2011). Las propiedades texturales del TPA tienen relación con el análisis sensorial de las productos evaluados, por lo cual, se recomienda, en futuras investigaciones, aplicar pruebas sensoriales, para comprobar los resultados obtenidos.

Agradecimientos: Los autores agradecen a la empresa Ingredion de Colombia S.A., por facilitar la materia prima para realizar la investigación. Conflictos de interés: El manuscrito fue preparado y revisado con la participación de todos los autores, quienes declaramos que no existe ningún conflicto de interés que ponga en riesgo la validez de los resultados presentados. Financiación: Este estudio fue financiado por la Universidad Nacional de Colombia, sede Medellín. 


\section{BIBLIOGRAFÍA}

1. AMERICAN ASSOCIATION OF CEREAL CHEMISTS -AACC-. 2000. Approved Methods of Analysis, $10^{\text {th }}$ ed. Methods 44-15A, 74-09. American Association of Cereal Chemists. AACC International, St Paul, MN, USA.

2. AOAC INTERNATIONAL. 1997. Official Methods of Analysis, $16^{\text {th }}$ ed. Association of Official Analytical Chemists, Gaithersburg, MD, USA.

3. ANDERSON, R.A.; CONWAY, V.F.; PFEIFER, V.F.; GRIFFIN, E.L. 1969. Gelatinization of corn grits by roll and extrusion - cooking. Cereal Sci. Today. 14:4-12.

4. ARENDT, E.K.; MORRISSEY, A.; MOORE, M.M.; DAL BELLO, F. 2008. 13 - Gluten-free breads. In: Elke, K.A.; Dal Bello, F. (eds). Gluten-Free Cereal Products and Beverages. Ed. Academic Press. p.289-319.

5. CAPPA, C.; LUCISANO, M.; MARIOTTI, M. 2013. Influence of Psyllium, sugar beet fibre and water on gluten-free dough properties and bread quality. Carbohydr. Polym. 98(2):1657-1666.

6. CONFORTI, F.D.; DAVIS, S.F. 2006. The effect of soya flour and flaxseed as a partial replacement for bread flour in yeast bread. Int. J. Food Sci. Tech. 41:95101.

7. DE LA HERA, E.; ROSELL, C.M.; GOMEZ, M. 2014. Effect of water content and flour particle size on gluten-free bread quality and digestibility. Food Chem. 151:526-531.

8. DEMIRKESEN, I.; KELKAR, S.; CAMPANELLA, O.H.; SUMNU, G.; SAHIN, S.; OKOS, M. 2014. Characterization of structure of gluten-free breads by using X-ray microtomography. Food Hydrocoll. 36:37-44.

9. DEMIRKESEN, I.; MERT, B.; SUMNU, G.; SAHIN, S. 2010. Rheological properties of gluten-free bread formulations. J. Food Eng. 96(2):295-303.

10. DIOP, C.I.K.; LI, H.L.; XIE, B.J.; SHI, J. 2011. Effects of acetic acid/acetic anhydride ratios on the properties of corn starch acetates. Food Chem. 126(4):16621669.

11. GOESAERT, H.; BRIJS, K.; VERAVERBEKE, W.S.; COURTIN, C.M.; GEBRUERS, K.; DELCOUR, J.A. 2005. Wheat flour constituents: how they impact bread quality, and how to impact their functionality. Trends Food Sci. Tech. 16(1-3):12-30.
12. GUARDA, A.; ROSELL, C.M.; BENEDITO, C.; GALOTTO, M.J. 2004. Different hydrocolloids as bread improvers and antistaling agents. Food Hydrocoll. 18(2):241-247.

13. HAGER, A.S.; ARENDT, E.K. 2013. Influence of hydroxypropylmethylcellulose (HPMC), xanthan gum and their combination on loaf specific volume, crumb hardness and crumb grain characteristics of glutenfree breads based on rice, maize, teff and buckwheat. Food Hydrocoll. 32(1):195-203.

14. HASJIM, J.; LI, E.; DHITAL, S. 2013. Milling of rice grains: Effects of starch/flour structures on gelatinization and pasting properties. Carbohydr. Polym. 92(1):682-690.

15. INGREDION COLOMBIA S.A. 2013. ExpandexTM modified tapioca starch. Consultado 25 de mayo de 2013. Disponible desde Internet en: www.expandexglutenfree.com (con acceso 25/05/2013).

16. INTERNATIONAL ORGANIZATION FOR STANDARDIZATION -ISO-. 1997. ISO 10520: Determination of starch content - Ewers polarimetric method. International Organization for Standardization. Geneva, Switzerland.

17. JI, Y.; ZHU, K.; QIAN, H.; ZHOU, H. 2007. Staling of cake prepared from rice flour and sticky rice flour. Food Chem. 104(1):53-58.

18. KAUSHAL, P.; KUMAR, V.; SHARMA, H.K. 2012. Comparative study of physicochemical, functional, antinutritional and pasting properties of taro (Colocasia esculenta), rice (Oryza sativa) flour, pigeonpea (Cajanus cajan) flour and their blends. LWT - Food Sci. Tech. 48(1):59-68.

19. LAZARIDOU, A.; DUTA, D.; PAPAGEORGIOU, M.; BELC, N.; BILIADERIS, C.G. 2007. Effects of hydrocolloids on dough rheology and bread quality parameters in gluten-free formulations. J. Food Eng. 79(3):10331047.

20. LEE, S.Y.; CHEN, H.; HANNA, M.A. 2008. Preparation and characterization of tapioca starch-poly(lactic acid) nanocomposite foams by melt intercalation based on clay type. Indust. Crops Products. 28(1):95-106.

21. LÓPEZ-TENORIO, J.A.; RODRÍGUEZ-SANDOVAL, E.; SEPÚLVEDA-VALENCIA, J.E. 2012. Evaluación de caracteristicas fisicas y texturales de pandebono. Acta Agron. 61(3):273-281. 
22. MAHMOUD, R.M.; YOUSIF, E.I.; GADALLAH, M.G.E.; ALAWNEH, A.R. 2013. Formulations and quality characterization of gluten-free Egyptian balady flat bread. Ann. Agr. Sci. 58(1):19-25.

23. MANJARRES-PINZON, K.; CORTES-RODRIGUEZ, M.; RODRÍGUEZ-SANDOVAL, E. 2013. Effect of drying conditions on the physical properties of impregnated orange peel. Braz. J. Chem. Eng. 30(3):667-676.

24. MARIOTTI, M.; PAGANI, M.A.; LUCISANO, M. 2013. The role of buckwheat and HPMC on the breadmaking properties of some commercial gluten-free bread mixtures. Food Hydrocoll. 30(1):393-400.

25. MOHAMMADI, M.; SADEGHNIA, N.; AZIZI, M.H.; NEYESTANI, T.R.; MORTAZAVIAN, A.M. 2013. Development of gluten-free flat bread using hydrocolloids: Xanthan and CMC. J. Indust. Eng. Chem. 20(4):1812-1818.

26. ONYANGO, C.; MUTUNGI, C.; UNBEHEND, G.; LINDHAUER, M.G. 2009a. Creep-recovery parameters of gluten-free batter and crumb properties of bread prepared from pregelatinised cassava starch, sorghum and selected proteins. Int. J. Food Sci. Tech. 44:2493-2499

27. ONYANGO, C.; UNBEHEND, G.; LINDHAUER, M.G. 2009b. Effect of cellulose-derivatives and emulsifiers on creep-recovery and crumb properties of glutenfree bread prepared from sorghum and gelatinised cassava starch. Food Res. Int. 42(8):949-955.

28. ONYANGO, C.; UNBEHEND, G.; LINDHAUER, M. 2011. Modification of gluten-free sorghum batter and bread using maize, potato, cassava or rice starch. LWT Food Sci. Tech. 44(3):681-686.

29. PONGJARUVAT, W.; METHACANON, P.; SEETAPAN, N.; FUONGFUCHAT, A.; GAMONPILAS, C. 2014. Influ- ence of pregelatinised tapioca starch and transglutaminase on dough rheology and quality of gluten-free jasmine rice breads. Food Hydrocoll. 36:143-150.

30. RODRÍGUEZ, P.; SAN MARTÍN, M.E.; GONZÁLEZ DE LA CRUZ, G. 2001. Calorimetría diferencial de barrido y rayos-x del almidón obtenido por nixtamalización fraccionada. Superf. Vacío. 13:61-65.

31. RODRIGUEZ-SANDOVAL, E.; SANDOVAL, G.; CORTES-RODRÍGUEZ, M. 2012. Effect of quinoa and potato flours on the thermomechanical and breadmaking properties of wheat flour. Braz. J. Chem. Eng. 29(3):503-510.

32. RODRIGUEZ-SANDOVAL, E.; FRANCO, C.M.L.; MANJARRES-PINZON, K. 2014. Effect of fructooligosaccharides on the physicochemical properties of sour cassava starch and baking quality of gluten-free cheese bread. Starch-Stärke. 66:678-684.

33. SAHIN, S.; SUMNU, S.G. 2006. Physical Properties of Foods. Ed. Springer Verlag. New York, NY, USA. p.30-33.

34. SCIARINI, L.S.; RIBOTTA, P.D.; LEÓN, A.E.; PÉREZ, G.T. 2012. Incorporation of several additives into gluten free breads: Effect on dough properties and bread quality. J. Food Eng. 111(4):590-597.

35. ZIOBRO, R.; WITCZAK, T.; JUSZCZAK, L.; KORUS, J. 2013. Supplementation of gluten-free bread with non-gluten proteins. Effect on dough rheological properties and bread characteristic. Food Hydrocoll. 32(2):213-220.

Recibido: Noviembre 28 de 2014

Aceptado: Julio 21 de 2015

\section{Cómo citar:}

Martínez-Jiménez, F.; Rodríguez-Sandoval, R.; Hernández-Gómez, M.S. 2015. Impacto de la adición de caboximetilcelulosa y agua en las propiedades fisicoquímicas y de calidad de pan libre de gluten. Rev. U.D.C.A Act. \& Div. Cient. 18((2): 445-454. 